\title{
The synergistic effects of Apatinib combined with cytotoxic chemotherapeutic agents on gastric cancer cells and in a fluorescence imaging gastric cancer xenograft model
}

This article was published in the following Dove Press journal:

OncoTargets and Therapy

\author{
Jiuhuan Feng' \\ Shukui Qin ${ }^{2}$ \\ 'Institute of First Clinical Medicine, \\ Nanjing University of Chinese \\ Medicine, Nanjing, China; ${ }^{2}$ Oncology \\ Center of PLA, 8I Hospital Affiliated \\ to Nanjing University of Chinese \\ Medicine, Nanjing, China
}

\begin{abstract}
Introduction: Methylsulfonic apatinib (hereinafter referred to as Apatinib) is a small-molecule angiogenesis inhibitor highly and selectively targeted to vascular endothelial growth factor receptor-2. At present, a series of basic and clinical studies have confirmed that Apatinib monotherapy can inhibit the growth of different carcinomas. Our experiment aimed to determine whether there is a synergistic effect between the combination of the traditional cytotoxic chemotherapy drugs paclitaxel (TAX), oxaliplatin (L-OHP), 5-fluorouracil (5-FU), and Apatinib. Materials and methods: We evaluated the combined effect using cytological experiments and a fluorescence imaging xenograft model. In vitro, the inhibition of cell proliferation increased notably when Apatinib was combined with TAX, L-OHP, and 5-FU. Then, for the mechanistic research, we selected the optimal dose of drugs that also had a synergistic effect. Apatinib combined with the aforementioned drugs, especially the combination of Apatinib and 5-FU, decreased the invasion and migration ability of the cells and increased the apoptosis ratio; expression of the anti-apoptotic protein Bcl-2 significantly decreased, and expression of the pro-apoptotic protein Bax increased. In vivo, when Apatinib was combined with TAX, L-OHP, and 5-FU, the volume of the xenograft model was significantly inhibited, the strength of the green fluorescence was weakened and the microvessel density decreased.
\end{abstract}

Results: The combination of Apatinib with TAX and 5-FU was synergistic (coefficient of drug interaction $<1$ ); the combination effect of Apatinib and L-OHP was only additive, with a shorter associated survival time.

Conclusion: The combination of Apatinib and classical chemotherapy drugs may be an optimal choice for gastric cancer treatment.

Keywords: gastric cancer, Apatinib, chemotherapy, fluorescence imaging

\section{Introduction}

Gastric cancer is one of the most common malignant tumors of the digestive tract, and the morbidity and mortality rates are extremely high. Worldwide, there are 950,000 new gastric cancer cases every year, and the morbidity rate is ranked sixth; 720,000 patients died, and the mortality rate was ranked third. ${ }^{1,2}$ It was expected that there would be 670,000 new cases in 2015 , with 490,000 patient deaths, seriously threatening people's lives and health. ${ }^{3}$ At present, systemic chemotherapy or symptomatic supportive treatment are still the main choices for most gastric cancer patients; chemotherapy regimens, ${ }^{4-6}$ including platinum, fluorouracil, and taxane are confirmed to confer the best survival benefit as first-line treatments. ${ }^{7,8}$ Although the survival time
Correspondence: Shukui Qin Oncology Center of PLA, 8I Hospital Affiliated to Nanjing University of Chinese Medicine, No. 34 Changfu Street, Qinhuai District, Nanjing, Jiangsu 210002, China

Email qinsk@csco.org.cn 
was prolonged in the combined regimen, the median overall survival time was still shorter than 12 months, ${ }^{9,10}$ and there was more associated toxicity and side effects.

In 2014, the small-molecule tyrosine kinase inhibitor (TKI) Apatinib prolonged the survival time of gastric cancer patients by 1.8 months in a Phase III clinical trial, ${ }^{11}$ showing the importance of the anti-angiogenesis treatment for gastric cancer. Based on the result, Apatinib was approved by the China Food and Drug Administration for the third-line treatment of advanced gastric or gastroesophageal junction cancer. Thus far, many case reports ${ }^{12-16}$ confirm the clinical benefit of Apatinib in prolonging survival time and improving the clinical syndrome and show its advantage in real, clinical, and therapeutic environments.

The clinical benefit of anti-angiogenesis drugs has been limited, ${ }^{17}$ as has been the overall response rate; thus, combining Apatinib with chemotherapy will be the trend for antiangiogenesis treatments in the future. The LUME-lung 1 study, ${ }^{18}$ which was published in The Lancet in 2014, proved that anti-angiogenic TKIs combined with chemotherapy could provide clinical benefit for non-small cell lung cancer patients who failed in the first-line chemotherapy. Until now, there was no more powerful clinical evidence for the combination effect of Apatinib and chemotherapy. Therefore, we designed this experiment to test the combination effect using fundamental research, providing a reference for human subject research and personalized medicine in the future.

\section{Materials and methods}

\section{Equipment and reagents}

The equipment and reagents are as follows: gel imaging system (Gel Doc XR, Bio-Rad Laboratories Inc., Hercules, CA, USA), PowerPac ${ }^{\mathrm{TM}}$ Basic electrophoresis apparatus (1645051, Bio-Rad), gel imaging analysis system (SYNGENE G: BOXChemiXR5), microplate reader (680, Bio-Rad), flow cytometry (FACSCalibur, Becton-Dickinson, Franklin Lakes, NJ, USA), FLUOR-100 live animal fluorescence imaging system; Roswell Park Memorial Institute (RPMI)-1640 medium, fetal bovine serum (FBS; HyClone, Logan, UT, USA), MTT (Sigma-Aldrich Co., St Louis, MO, USA), PI (Sigma), annexin V-APC/7-AAD Cell Apoptosis Detection Kit (KaiJi Biology Co. Nanjing, Jiangsu, China), Bcl-2 monoclonal antibody, Bax monoclonal antibody, cleaved caspase-3 (c-caspase-3) antibody (CST Co., Louisville, KY, USA), polyvinylidene fluoride (PVDF) membrane (Merck Millipore, Billerica, MA, USA), green fluorescent protein (GFP) lentivirus (YuanDuan Biotechnology Co., Nanjing, China), polybrene application liquid (No: H9628, Sigma), and protein quantitative detection kit (Sigma).

The drugs for the in vitro experiment included paclitaxel lyophilized powder (A0820A), oxaliplatin lyophilized powder
(O0701A), fluorouracil lyophilized powder (D0405A) (all bought from MeiLun Biopharmaceutical Company, Dalian, Shandong, China), and Apatinib (purity $\geq 99 \%$ ) (Jiangsu HengRui Pharmaceutical Co., Jiangsu, China). The drugs for the in vivo experiment were paclitaxel injection(Sichuan TaiJi Pharmaceutical Co., ChongQin, China, No: 1412009, 30 mg/5 mL), oxaliplatin injection (Jiangsu HengRui Pharmaceutical Co., No: 15091416, $50 \mathrm{mg}$ ), fluorouracil injection (Tianjin JinYao Pharmaceutical Co., TianJin, China, No: 1508291, $0.25 \mathrm{~g} / 10 \mathrm{~mL}$ ), and Apatinib (purity $\geq 99 \%$ ) (Jiangsu HengRui Pharmaceutical Co. Ltd). Drugs were abbreviated as follows: paclitaxel, TAX; oxaliplatin, L-OHP; fluorouracil, 5-FU.

\section{The cell lines and xenograft model}

Human gastric adenocarcinoma cell lines SGC-7901 and BGC-823 (cell bank of Chinese Academy of Science, Shanghai, China) were approved by the ethics committee. BALB/c nude mice, female, specific pathogen-free (SPF) level, 5 weeks old were used for the xenograft model (Yangzhou University Comparative Medical Center, Yangzhou, China, animal certificate number: NO 201600829 , production license number: SCXK Su 20120004). The cells were cultured in RPMI-1640 medium (10\% FBS, $100 \mathrm{U} / \mathrm{mL}$ penicillin, and $100 \mathrm{U} / \mathrm{mL}$ streptomycin) and placed in an incubator at $37^{\circ} \mathrm{C}$ and $5 \% \mathrm{CO}_{2}$. Animals were kept and tested in the SPF barrier system, following the rules of Nanjing University of Traditional Chinese Medicine animal management and with approval from the animal ethics committee. This study was approved by the Institutional Animal Care and Use Committee of Nanjing University of Chinese Medicine, and all procedures were conducted in accordance with the guidelines of the National Institutes of Health Animal Care and Use Committee.

\section{Western blot assay}

After removing the cell culture medium, cold PBS was added, and the cells were washed twice; then, an appropriate amount of trypsin digestion solution was added. Cells were then centrifuged at 1,000 rpm for $10 \mathrm{~min}$. Twice, cold PBS was added, and cells were centrifuged at 1,000 rpm for $5 \mathrm{~min}$ for washing. Then, $10 \mu \mathrm{L}$ of phosphatase inhibitor, $1 \mu \mathrm{L}$ of protease inhibitor, and $5 \mu \mathrm{L}$ of $100 \mathrm{mM}$ phenylmethylsulfonyl fluoride were added to $1 \mathrm{~mL}$ of cold lysis buffer. After incubating cells on ice for several minutes, $1 \mathrm{~mL}$ of wellprepared cold lysis buffer was added to the cells, which were then shaken at $4^{\circ} \mathrm{C}$ for $15 \mathrm{~min}$. The supernatant was taken to quantify the protein using the Bradford method (Sigma). Sodium dodecyl sulfate polyacrylamide gel electrophoresis separation gel and concentration gels were prepared; then, the 
appropriate amount of sample was added to the gel. The protein ladder was added to the adjacent well, and electrophoresis began. Electrophoresis stopped when the target band had run $2 / 3$ of the way down the gel; then, proteins were transferred to PVDF membranes. After sealing the PVDF membrane, the primary antibody was added, followed by the secondary antibody. Membranes were washed thoroughly, and the ECL chemiluminescence kit was added (Pierce Bio, Rockford, IL, USA). Protein bands were visualized using G: BOX chemiXR5, and the data were analyzed using Gel-Pro32 software (Media Cybernetics, Rockville, MD, USA).

\section{Cytotoxicity assay}

The cytotoxicities of Apatinib and 3 chemotherapeutic drugs (TAX, L-OHP, and 5-FU) for 2 differentiated gastric cancer cell lines (BGC-823 and SGC-7901) were determined by MTT assay. Cells were incubated for $24 \mathrm{~h}$ $\left(37^{\circ} \mathrm{C}, 5 \% \mathrm{CO}_{2}\right)$ and divided into 8 groups (cell density: 4.0 $\times 10^{4} /$ per well): 1) Control; 2) Apatinib (4 $\left.\mu \mathrm{g} / \mathrm{mL}\right)$; 3) Apatinib (8 $\mu \mathrm{g} / \mathrm{mL})$; 4) Apatinib $(16 \mu \mathrm{g} / \mathrm{mL})$; 5) TAX/LOHP/5-FU; 6) (TAX/L-OHP/5-FU)+Apatinib (4 $\mu \mathrm{g} / \mathrm{mL})$; 7) $(\mathrm{TAX} / \mathrm{L}-\mathrm{OHP} / 5-\mathrm{FU})+$ Apatinib $(8 \mu \mathrm{g} / \mathrm{mL})$; and 8$)(\mathrm{TAX} /$ L-OHP/5-FU)+Apatinib $(16 \mu \mathrm{g} / \mathrm{mL})$. First, $100 \mu \mathrm{L}$ of drug was added into each well for $24 \mathrm{~h}(0.9 \%$ saline for the control group), and $20 \mu \mathrm{L}$ of MTT was added into each well for another $4 \mathrm{~h}$. After removing the culture medium, $150 \mu \mathrm{L}$ of dimethyl sulfoxide was added, and the 96-well flat plates were shaken; the optical density (OD) value at $490 \mathrm{~nm}$ was tested in an enzyme-labeled meter. The cell survival rate $(\%)=\mathrm{OD}$ value of the experimental group/OD value of the control group $\times 100 \%$. Each measurement was repeated 3 times, and the average was taken. To choose the proper drug concentration (cell survival rate remained as $85 \%-90 \%$ ) for the following mechanism study, the combination effect was analyzed with coefficient of drug interaction (CDIz0 value: $\mathrm{CDI}=\mathrm{E}_{\mathrm{AB}} /\left(\mathrm{E}_{\mathrm{A}} \times \mathrm{E}_{\mathrm{B}}\right)$ when $\mathrm{E}_{\mathrm{AB}}$ is the $\mathrm{OD}$ value ratio of the combination group and control group, $\mathrm{E}_{\mathrm{A}}$ is the $\mathrm{OD}$ value ratio of drug $\mathrm{A}$ and control group, and $\mathrm{E}_{\mathrm{B}}$ is the $\mathrm{OD}$ value ratio of drug $\mathrm{B}$ and control group. If $\mathrm{CDI}<1$, the 2 drugs combined were synergistic; $\mathrm{CDI}=1$, the 2 drugs combined were additive; $\mathrm{CDI}>1$, the 2 drugs combined were antagonistic. ${ }^{19}$

\section{Apoptosis was detected by flow cytometry}

The cells in the logarithmic growth phase were digested and adjusted to a density of $4 \times 10^{5} / \mathrm{mL}$. Then, the cells were divided into 8 groups after $12 \mathrm{~h}$ incubation (according to the result of the cytotoxicity assay, $4 \mu \mathrm{g} / \mathrm{mL}$ Apatinib was appropriate for the next step assay): 1) Control; 2) Apatinib; 3) TAX; 4) $\mathrm{TAX}+$ Apatinib; 5) L-OHP; 6) L-OHP+Apatinib; 7) 5-FU; and 8) 5-FU+Apatinib. The cells were washed and centrifuged after $24 \mathrm{~h}$ incubation with the drugs $(2,000 \mathrm{rpm}, 5 \mathrm{~min})$, and the single cell suspension was fixed in $70 \%$ ethanol for $2 \mathrm{~h}$. After centrifuging the cells and removing the supernatant, $500 \mu \mathrm{L}$ of binding buffer, $5 \mu \mathrm{L}$ of annexin V-APC, and $5 \mu \mathrm{L}$ of 7-AAD were mixed and added to the cells. The reaction proceeded for $15 \mathrm{~min}$, and then, apoptosis was tested.

\section{Transwell invasion and migration assay}

First, $50 \mu \mathrm{L}$ of $100 \mu \mathrm{g} / \mathrm{mL}$ matrigel was added to the upper chamber (Millipore) and saved for the following assay. Cells were cultured without serum for $12 \mathrm{~h}$ and then inoculated in the seasoned chamber after adding the warm media without serum to hydrate the basement membrane. The medium was removed $30 \mathrm{~min}$ later, and $200 \mu \mathrm{L}$ of the cell suspension (cell density: $5.0 \times 10^{5} / \mathrm{mL}$ ) and different drugs were added. Then, $500 \mu \mathrm{L}$ of medium with serum was added to the lower chamber and cultured for another $24 \mathrm{~h}$. The chambers were seasoned and fixed by $95 \%$ ethanol after removing the matrigel and liquid. The cells in the chamber were dyed with $0.1 \%$ crystal violet. Then, 5 visual fields at $100 \times$ were selected using an inverted microscope, and the cells were counted using Image-Pro Plus software (Media Cybernetics). The average number of the 5 fields was marked as the number of permeated cells in the invasion assay. The migration assay used a similar procedure, but a serum-free medium was used, and the cell density was adjusted to $1.0 \times 10^{6} / \mathrm{mL}$.

\section{GFP transfection assay}

After digesting the cells and adjusting the cell density to $1.0 \times 10^{5} /$ per well, polybrene was prepared, and the concentration was adjusted to $50 \mu \mathrm{g} / \mathrm{mL}$. The virus titer was $1.0 \times 10^{8} \mathrm{TU} / \mathrm{mL}$. Virus was used to infect the BGC-823 cells in groups ( 2 copies in every group). The virus-containing medium was removed and changed to RPMI-1640 culture medium with $10 \%$ FBS. The GFP expression after $48 \mathrm{~h}$ was observed using a fluorescence microscope. Cells were incubated in media containing G418 at different concentrations $(200-1,000 \mu \mathrm{g} / \mathrm{mL})$. Stable passage with obvious GFP expression in $95 \%$ of cells was considered a successful transfection. In the end, we obtained the GFP-transfected cells: BGC-823/GFP.

\section{The establishment of a nude mouse tumor model in fluorescence imaging and pharmacodynamics experiments}

The BGC-823/GFP cells were cultured and passaged to logarithmic growth phase. The cells were digested to obtain $0.2 \mathrm{~mL}$ cell suspension (cell density: $5.0 \times 10^{6} / \mathrm{mL}$ ). The suspension 
was subcutaneously implanted into the right axillary sides of the nude mice. The whole procedure was repeated, and the third generation was obtained, randomly grouping the mice by mouse weight $(\mathrm{n}=6)$. The groups were as follows: single-drug pharmacodynamic experiment groups: 1) Control; 2) Apatinib $37.5 \mathrm{mg} / \mathrm{kg}$; 3) Apatinib $75 \mathrm{mg} / \mathrm{kg}$; and 4) Apatinib $150 \mathrm{mg} / \mathrm{kg}$; combination drug pharmacodynamic experiment groups: 1) Control; 2) Apatinib; 3) TAX; 4) TAX+Apatinib; 5) L-OHP; 6) L-OHP+Apatinib; 7) 5-FU; and 8) 5-FU+Apatinib. Dosage and mode of administration were as follows: TAX (10 mg/kg every 3 days, iv), L-OHP ( $10 \mathrm{mg} / \mathrm{kg}$ every 3 days, ip), 5 -FU (30 mg/kg every 3 days, ip), and Apatinib ( $75 \mathrm{mg} / \mathrm{kg}$ every day, po). The mice were medicated when tumor volumes reached $100-150 \mathrm{~mm}^{3}$, and the diet, mental state, and defecation conditions were recorded. The in vivo fluorescent images were obtained using the fluorescent system every 3 days (imaging conditions: bandpass filter: 445-490 nm, part N, M080283; exposure time for fluorescence imaging: 3,000 ms; detection wavelength setting of emission light: 500-650 nm; imaging software: Maestr). The weight was recorded, the major and minor axes of the tumor were measured every 2 days, and the weight curve was drawn. The tumor volume was calculated using Image-Pro.1 software $\left(\mathrm{V}=\mathrm{L} \times \mathrm{W}^{2} / 2\right)$ to evaluate the antineoplasmic activity of single drug and combination.

The antineoplasmic activity was evaluated by the relative tumor proliferation rate: $\mathrm{T} / \mathrm{C}(\%)=\mathrm{TRTV} / \mathrm{CRTV} \times 100 \%$ where $\mathrm{RTV}=\mathrm{Vt} / \mathrm{Vo}$, Vt is the tumor volume after medication, Vo is the tumor volume before medication, TRTV means the RTV for the drugs, and CRTV means the RTV for the control. $\mathrm{T} / \mathrm{C} \% \leq 60 \%$ and $P<0.05$ were considered to be effective; $\mathrm{T} / \mathrm{C}>60 \%$ was considered as invalid. The tumor growth inhibition rate $(\mathrm{IR} \%)=($ average tumor weight in the control group-average tumor weight in the medicated group)/average tumor weight in the control group $\times 100 \%$. Regarding the results, IR $<40 \%$ means invalid; $\geq 40 \%$ and $P<0.05$ means effective. The results were analyzed by the combination effect index $(\mathrm{CDI})=\mathrm{E}_{\mathrm{AB}} /\left(\mathrm{E}_{\mathrm{A}} \times \mathrm{E}_{\mathrm{B}}\right)$, where $\mathrm{E}_{\mathrm{AB}}$ is the ratio of the tumor weight of the combination group to that of the control group, $\mathrm{E}_{\mathrm{A}}$ is the ratio of the tumor weight of the A group to the control group, and $\mathrm{E}_{\mathrm{B}}$ is the ratio of the tumor weight of the B group to the control group. Regarding the drug combinations, $\mathrm{CDI}<1$ means synergistic, $\mathrm{CDI}=1$ means additive, and $\mathrm{CDI}>1$ means antagonistic. In addition, 6 additional mice in each group were tested for the survival assay, with 7 days rest after 14 days of medication constituting 1 cycle. The cycle was repeated 3 times, and the survival time was observed.

\section{The microvessel density (MVD) assay}

Vascular endothelial cells were labeled with CD31 antibody, and the MVD was tested by an immunohistochemical method according to the Weidner counting criteria. The slices were viewed at low magnification $(40 \times)$ to observe the whole field and 3 regions that were rich in vascular tissue (brown staining region with highest density) were chosen. The microscope was adjusted to the highest magnification $(200 \times)$, and the microvessel numbers were counted in a double-blind manner. The average number was the MVD. ${ }^{20}$

\section{Statistical analysis}

SPSS 19.0 software was used to analyze the data (means \pm SD). Paired samples were analyzed by paired sample $t$-test, and multiple samples were analyzed by one-way analysis of variance; $P<0.05$ was statistically significant.

\section{Result}

\section{Apatinib as monotherapy and combined with chemotherapy drugs can inhibit the proliferation of gastric cancer cells}

Vascular endothelial growth factor receptor (VEGFR)-2 protein expression was detected in 3 human gastric cancer cells, and the expression of VEGFR-2 protein was negatively correlated with cell differentiation (Figure 1). Based on the preliminary experiment result, we set 6 dose levels for Apatinib monotherapy $(2.00,4.00,8.00,16.00,32.00$, and $64.00 \mu \mathrm{g} / \mathrm{mL}$ ). The results showed that the proliferation inhibition of Apatinib in the 2-cell lines was dose-dependent, with certain individual differences (Figure 1). We selected 4.00, 8.00, and $16.00 \mu \mathrm{g} / \mathrm{mL}$ as low, medium, and high levels of Apatinib in the following combination test. The proper doses for the 3 chemotherapy drugs, chosen according to the research literature results and in preliminary experiments, also had lower cytotoxicity $(\sim 90 \%$ of cells survive). The proliferation inhibition effect was notably strengthened when the chemotherapy drugs were combined with Apatinib in the 2 cells. The significant difference was significant $(P<0.05)$ when compared with that of the chemotherapy monotherapy group. According to our result, the synergistic effect was obvious in the Apatinib+TAX group, and individual differences were observed with the combination of Apatinib+L-OHP and Apatinib+5-FU. For the next mechanism experiment, we chose the BGC-823 cell line for its high and stable expression of VEGFR-2 protein; then, we selected $4 \mu \mathrm{g} / \mathrm{mL}$ Apatinib for the combination assays (Table 1). 
A
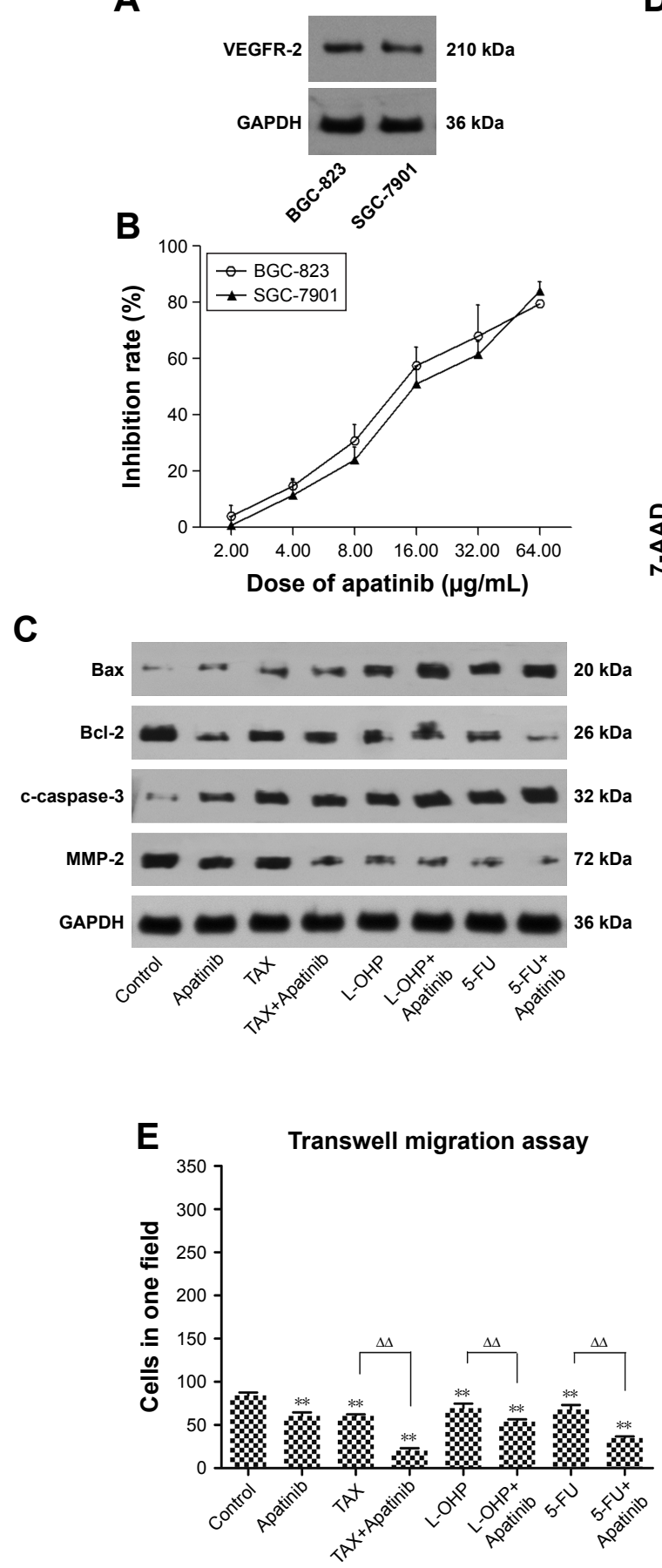
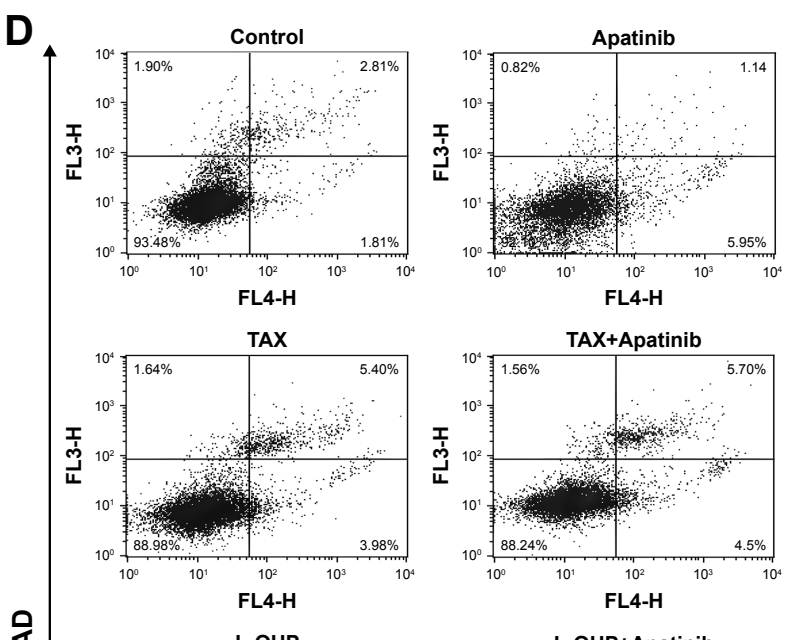

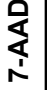
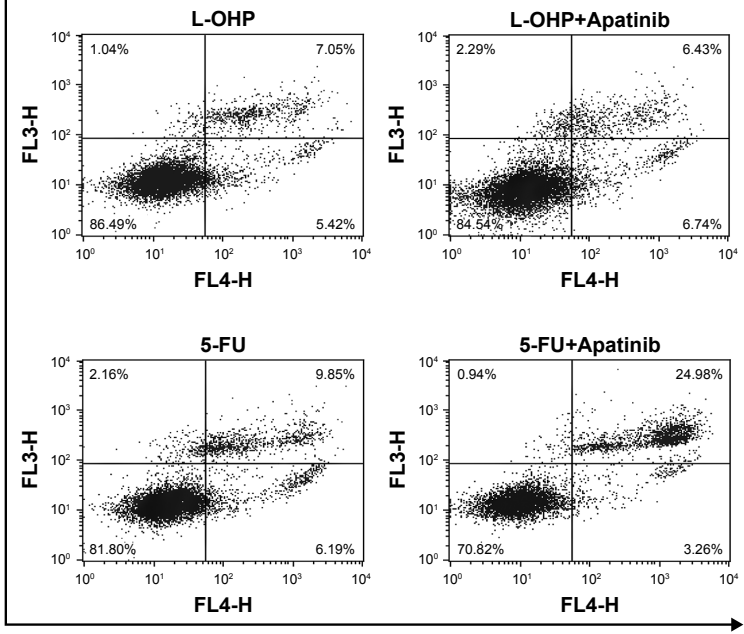

Annexin V-APC

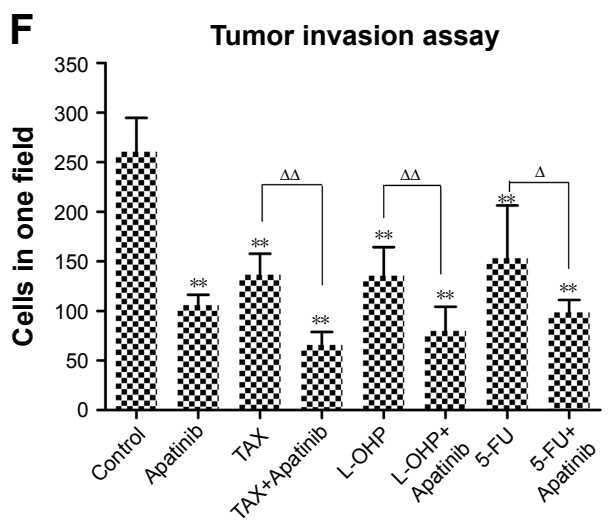

Figure I VEGFR-2 protein expression of the gastric cancer cells with different differentiation (A); the proliferation inhibition rate for Apatinib to 2 cell lines with different concentration (B); the Bcl-2, Bax, c-caspase-3 and MMP-2 protein expression of different groups (C); the apoptosis rate of different groups (D); the exact transmembrane cells of different groups in migration assay $(\mathbf{E})$ and in invasion assay $(\mathbf{F}) ; * * P<0.01$ medication group vs control group; $\Delta P<0.05$ combination group vs chemotherapy monotherapy group, ${ }^{\Delta} \mathrm{P}<0.0 \mathrm{I}$ combination group vs chemotherapy monotherapy group. Abbreviations: 5-FU, 5-fluorouracil; L-OHP, oxaliplatin; TAX, paclitaxel.

\section{Apatinib combined with chemotherapy promotes the apoptosis rate}

TAX, L-OHP, 5-FU, and Apatinib monotherapy increased the rate of cell apoptosis; the difference was significant compared with that of the control group $(P<0.05$; Figure 1).
The apoptosis rate increased in the 5-FU+Apatinib group and was much higher than the 5-FU group; the difference was significant $(P<0.05)$. The rate of apoptosis was not obviously increased in the TAX+Apatinib group and the L-OHP+Apatinib group $(P>0.05)$. The expression of 
Table I CDI for 2 cells

\begin{tabular}{|c|c|c|c|}
\hline & \multicolumn{3}{|l|}{ BGC-823 } \\
\hline & $\begin{array}{l}\text { TAX } \\
(3.75 \mathrm{ng} / \mathrm{mL})\end{array}$ & $\begin{array}{l}\text { L-OHP } \\
(1.00 \mu \mathrm{g} / \mathrm{mL})\end{array}$ & $\begin{array}{l}\text { 5-FU } \\
(0.25 \mu \mathrm{g} / \mathrm{mL})\end{array}$ \\
\hline Apatinib $(4.00 \mu g / m L)$ & 0.97 & 0.99 & 0.96 \\
\hline Apatinib $(8.00 \mu \mathrm{g} / \mathrm{mL})$ & 0.95 & 1.03 & 1.06 \\
\hline \multirow[t]{3}{*}{ Apatinib $(16.00 \mu \mathrm{g} / \mathrm{mL})$} & 0.91 & 1.05 & 1.07 \\
\hline & \multicolumn{3}{|l|}{ SGC-790I } \\
\hline & $\begin{array}{l}\text { TAX } \\
(3.75 \mathrm{ng} / \mathrm{mL})\end{array}$ & $\begin{array}{l}\text { L-OHP } \\
(0.50 \mu \mathrm{g} / \mathrm{mL})\end{array}$ & $\begin{array}{l}5-F U \\
(0.50 \mu \mathrm{g} / \mathrm{mL})\end{array}$ \\
\hline Apatinib $(4.00 \mu \mathrm{g} / \mathrm{mL})$ & 0.91 & 1.05 & 1.11 \\
\hline Apatinib $(8.00 \mu \mathrm{g} / \mathrm{mL})$ & 0.97 & 1.02 & 1.01 \\
\hline Apatinib $(16.00 \mu \mathrm{g} / \mathrm{mL})$ & 1.00 & 0.97 & 0.99 \\
\hline
\end{tabular}

Notes: $C D I=E_{A B} /\left(E_{A} \times E_{B}\right), E_{A B}$ is the $O D$ value ratio of combination group and control group, $E_{A}$ is the $O D$ value ratio of drug $A$ and control group, $E_{B}$ is the $O D$ value ratio of drug $B$ and control group. If $C D I<I$, the 2 drugs combined were synergistic; $C D I=I$, the 2 drugs combined were additive; $C D I>I$, the 2 drugs combined were antagonistic

Abbreviations: 5-FU, 5-fluorouracil; CDI, combination effect index; L-OHP, oxaliplatin; TAX, paclitaxel.

apoptosis-related proteins changed in the combination groups: the expression of anti-apoptosis protein $\mathrm{Bcl}-2$ decreased, and pro-apoptosis protein Bax and apoptotic effector c-caspase-3 increased at different levels (Figure 1).

\section{Apatinib combined with chemotherapy inhibits invasion and migration ability}

In the invasion assay, the number of invaded cells decreased in each monotherapy group and each combination group (Figure 1); statistical significance existed when the monotherapy groups were compared with the corresponding combination groups $(P<0.05)$. In the migration assay, we also observed the same result but at different levels (Figure 1). The expression of the protein MMP-2 was significantly decreased in the combination group; therefore, we hypothesize that the inhibition of the invasion and migration ability might have a relationship with the decreased expression of the protein MMP-2 (Figure 1).

\section{Apatinib inhibits the tumor growth of gastric cancer xenografts}

After the successful transfection of fluorescent protein, we saw strong green fluorescence expression of the cells in the microscope. At the first day of transplantation, we observed subcutaneous green fluorescent cells; with the growth of the tumor, the green fluorescence did not decline but was stably expressed. Based on the data from former literature about the maximum tolerated dose for beagles, ${ }^{21}$ we converted $30 \mathrm{mg} / \mathrm{kg}$ to obtain the high dose of Apatinib as $150 \mathrm{mg} / \mathrm{kg}$. Then, we determined the medium and low doses of Apatinib using the 0.5 geometric relation $(75$ and $37.5 \mathrm{mg} / \mathrm{kg}$, respectively). In the preliminary experiment, the high dose of Apatinib did not show obvious toxicity or lethality. In the formal experiment, we measured the tumor volume every 2 days. The amplification of the 3 Apatinib groups was small; also, a significant difference existed compared with that of the control group $(P<0.05)$, but the differences among the 3 Apatinib groups were not significant $(P<0.05$; Table 2$)$. The weight of the mice decreased after the medication by $1.2 \%$ for the control group, $16 \%$ for the low Apatinib group, $13.4 \%$ for the medium Apatinib group, and 16.8\% for the high Apatinib group. After the medication treatment, we sacrificed the mice and retrieved and weighed the tumors (Figure 2A). A statistical significance was found when comparing the 3 Apatinib groups with the control group $(P<0.05)$ and $\mathrm{IR} \geq 40 \%$.

\section{Apatinib combined with chemotherapy enhances the inhibition ability of tumor growth}

The activity of the mice decreased gradually in the first 2 weeks. Also, the reaction of the mice became slower in the medication group. The weights of the mice decreased at different levels. Furthermore, 1 mouse in the L-OHP group and 1 in the Apatinib group died in the last week of medication; 1 mouse in the L-OHP+Apatinib group died at the last day of the medication. Pathological sections were generated from the dead nude mice; in the liver, a slight inflammatory infiltration in the hepatic lobule and portal area, slight edema and steatosis in the hepatocytes, and mild expansion of hepatic sinusoid and extravasated blood were observed. In the kidney pathological sections, slight hydropic degeneration in the renal tubular epithelial cells, mild denaturation and necrosis in the renal tubules, and inflammatory infiltration in the mesenchyme were observed. Based on the preliminary experiment and pathological analysis, we concluded that slight toxicity occurred in the $\mathrm{L}-\mathrm{OHP}+$ Apatinib group.

Dynamic monitoring of the fluorescence image of each group was performed in the experiment (Figure 3). The green fluorescence was stably expressed, and the area of green fluorescence in the image increased slowly in the combination groups. We can assume that the tumor inhibition ability

Table 2 Pharmacodynamics experiment result for Apatinib monotherapy group

\begin{tabular}{llll}
\hline & $\begin{array}{l}\text { Apatinib } \\
(37.5 \mathrm{mg} / \mathrm{kg})\end{array}$ & $\begin{array}{l}\text { Apatinib } \\
\mathbf{( 7 5} \mathbf{~ m g} / \mathbf{k g})\end{array}$ & $\begin{array}{l}\text { Apatinib } \\
(150 \mathrm{mg} / \mathbf{k g})\end{array}$ \\
\hline $\mathrm{T} / \mathrm{C}(\%)$ & $47.82^{* *}$ & $46.50^{* *}$ & $36.38^{* *}$ \\
\hline
\end{tabular}

Note: $* * P<0.01$ medication group vs control group. 
A

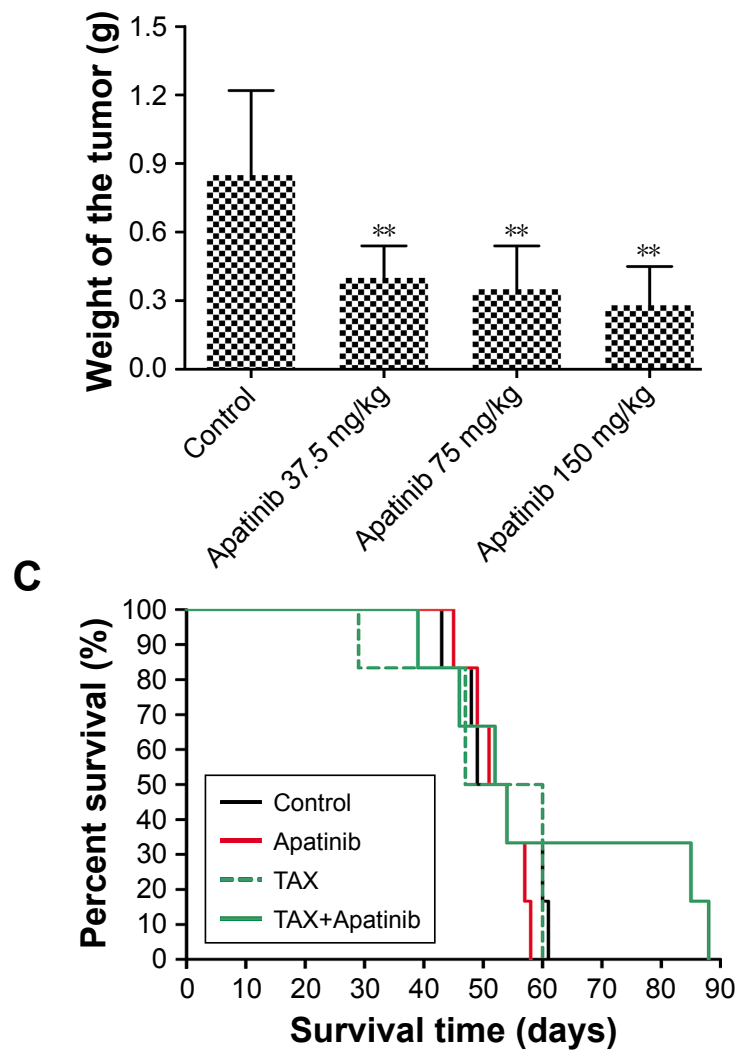

E

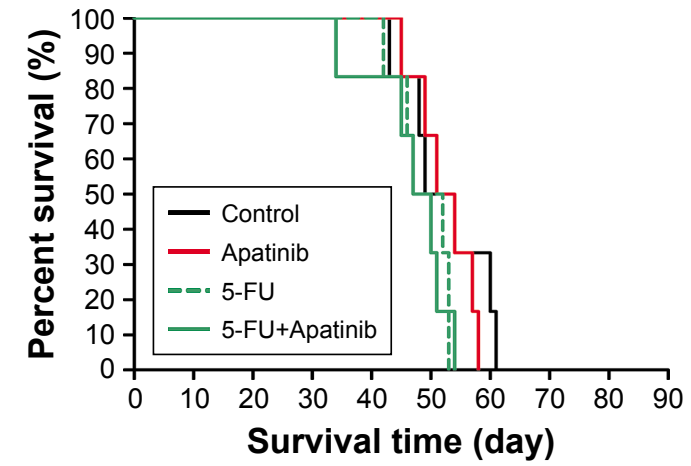

F

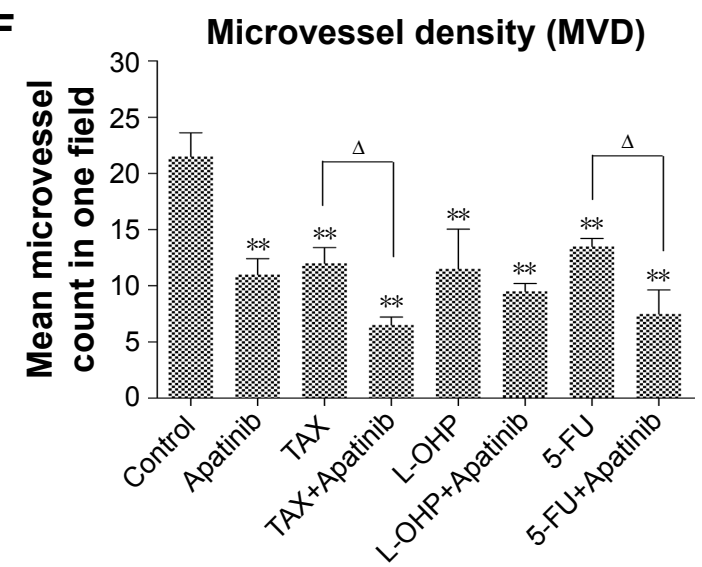

B

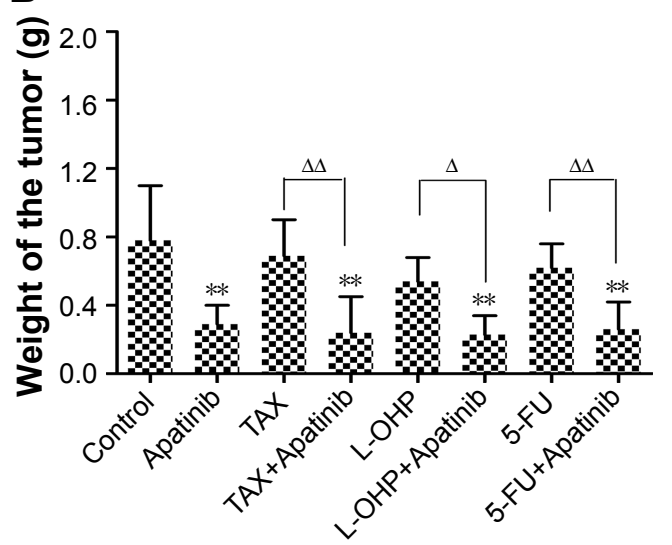

D

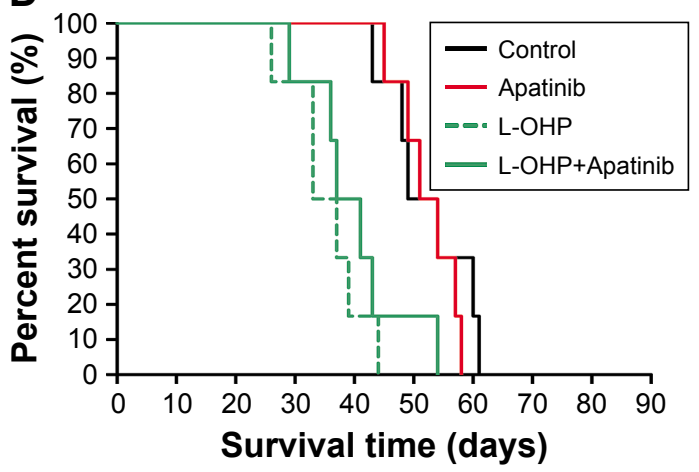

G

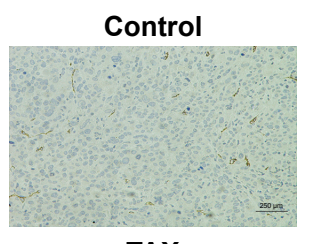

TAX

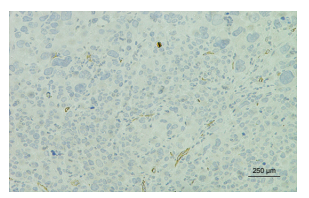

L-OHP

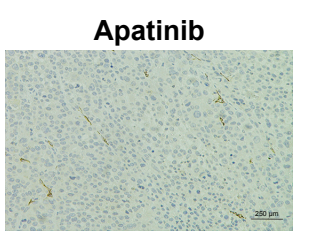

TAX+Apatinib

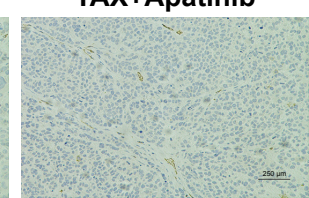

L-OHP+Apatinib

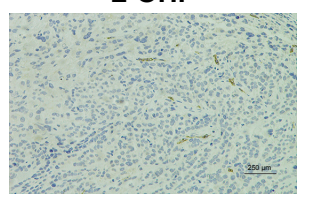

5-FU

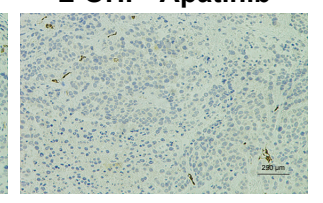

5-FU+Apatinib
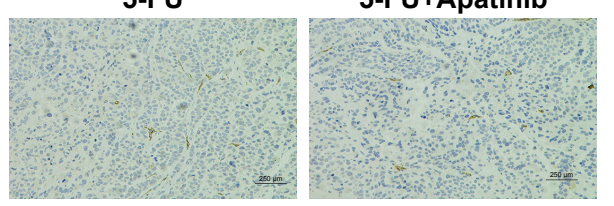

Figure 2 The tumor weight of Apatinib monotherapy groups $(\mathbf{A})$; the tumor weight of combination groups $(\mathbf{B}), * * P<0.01$ medication group vs control group, $\triangle P<0.05$ combination group vs chemotherapy monotherapy group, $\triangle P P<0.01$ combination group vs chemotherapy monotherapy group; the survival time for the combination of Apatinib with TAX (C), L-OHP (D), and 5-FU (E); the tumor microvessel images of different groups marked by CD3I antibody $(\mathbf{G})(\times 200$ times) and exactly the number of marked microvessel $(\mathbf{F}) ; * * P<0.01$ medication group vs control group; ${ }^{\Delta P}<0.05$ combination group vs chemotherapy monotherapy group, $\Delta \Delta P<0.01$ combination group vs chemotherapy monotherapy group.

Abbreviations: 5-FU, 5-fluorouracil; L-OHP, oxaliplatin; TAX, paclitaxel. 
A Image before the medication

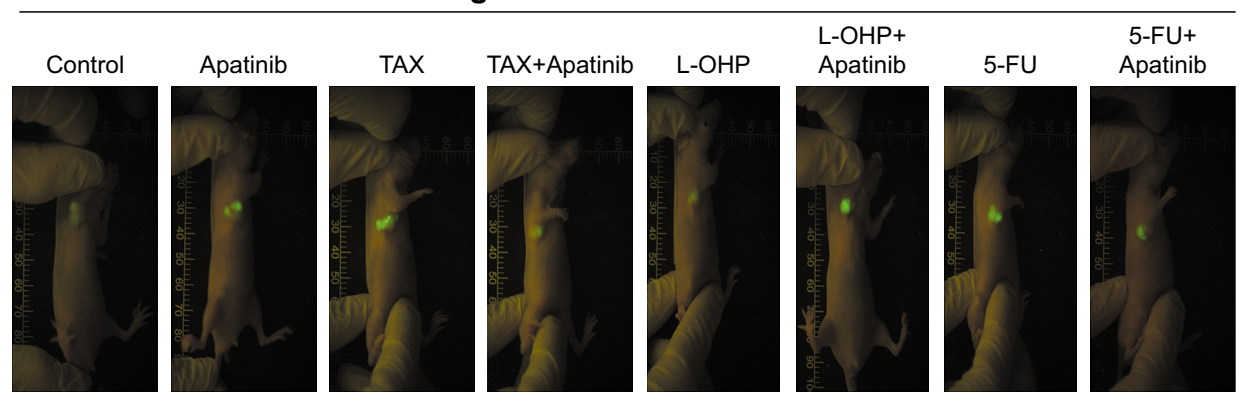

B Image during the medication

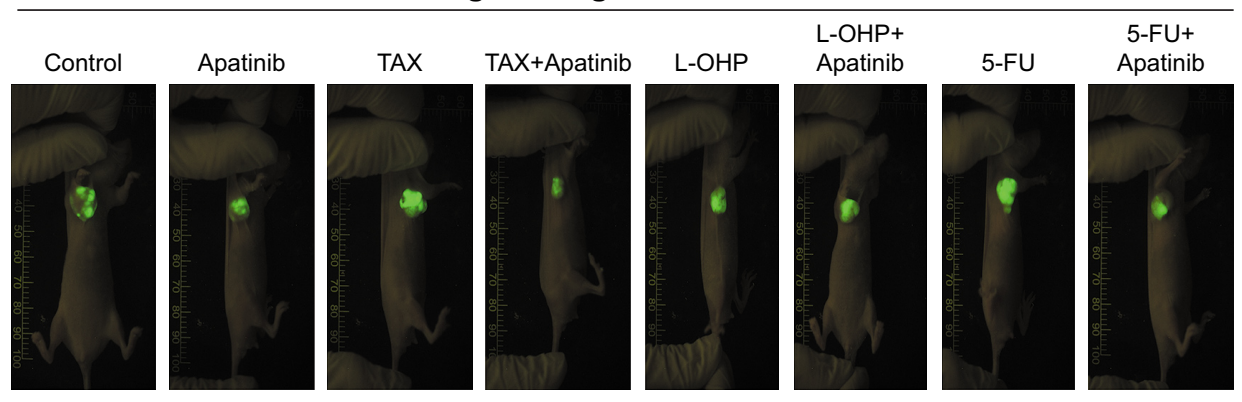

C

Image after the medication

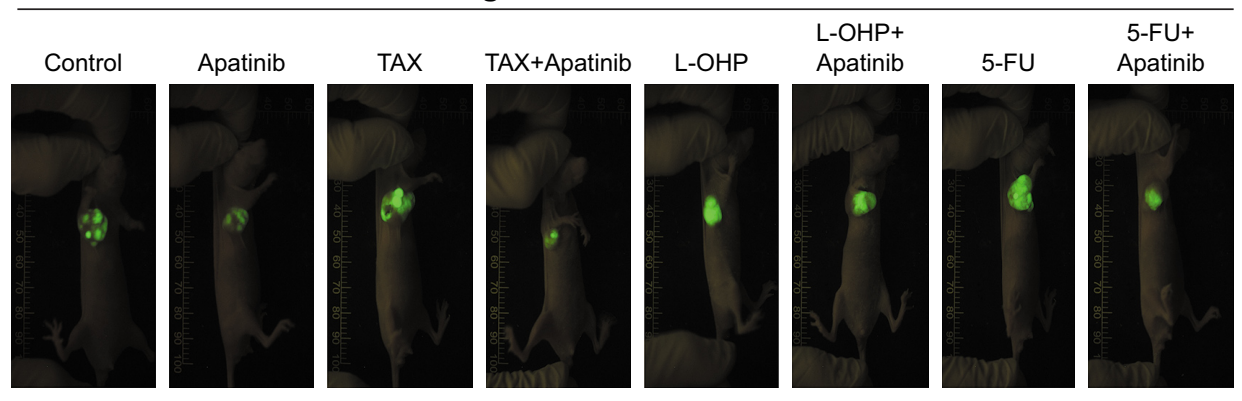

Figure 3 The dynamic fluorescence images of the tumor.

Abbreviations: 5-FU, 5-fluorouracil; L-OHP, oxaliplatin; TAX, paclitaxel.

increased when Apatinib was combined with TAX, L-OHP, and 5-FU, especially TAX and 5-FU. In the pharmacodynamic experiment, we measured the tumor volume every 2 days; the tumor volume amplification of the control group was obvious, and the comparisons between the control group and other groups, the combination groups and the chemotherapy monotherapy groups were also obvious $(P<0.05)$. The $\mathrm{T} / \mathrm{C}(\%)$ values were all $\leq 60 \%$ (Table 3 ). We assumed that the antitumor activity increased when Apatinib was combined with TAX, L-OHP, and 5-FU. The weight of mice in the L-OHP+Apatinib group notably decreased, while that of the other groups changed slightly. We analyzed the combination effect using the CDI index; CDI was $<1$ when TAX and 5-FU were combined with Apatinib and equal to 1 when L-OHP was combined with Apatinib. Therefore, the synergistic effect was obvious when TAX and 5-FU were combined with Apatinib but not obvious for the combination of L-OHP and Apatinib. This result was similar to the fluorescence imaging results. After the medication treatment, we sacrificed the mice and retrieved and weighed

Table 3 The pharmacodynamics results for the combination of Apatinib and chemotherapy drugs

\begin{tabular}{llllllll}
\hline Groups & Apatinib & TAX & T+A & L-OHP & L+A & 5-FU & F+A \\
\hline T/C $(\%)$ & $39.33^{* *}$ & $66.75^{*}$ & $33.09^{* *, \Delta}$ & $75.64^{*}$ & $29.19 * *, \Delta$ & 83.65 & $26.73^{* *, \Delta \Delta}$ \\
\hline
\end{tabular}

Notes: $* P<0.05$ medication group vs control group, ${ }^{*} P<0.01$ medication group vs control group; ${ }^{\triangle P}<0.05$ combination group vs chemotherapy group, $\triangle \Delta P<0.0$ I combination group vs chemotherapy group. T/C (\%): relative tumor proliferation rate (\%)/relative tumor volume for control group $\times 100 \%$; T+A: TAX + apatinib; L+A: L-OHP + apatinib; $\mathrm{F}+\mathrm{A}: 5-\mathrm{FU}+$ apatinib.

Abbreviations: 5-FU, 5-fluorouracil; L-OHP, oxaliplatin; TAX, paclitaxel. 
the tumors (Figure 2B). A statistical significance was found when comparing the 3 chemotherapy monotherapies with the combination groups $(P<0.05)$ and $\mathrm{IR} \geq 40 \%$.

\section{Apatinib combined with chemotherapy has a minor effect on the survival time}

The exact survival times of mice were as follows: control group,

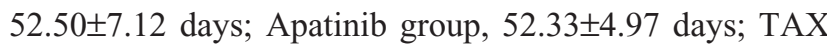
group, 50.50 \pm 12.31 days; L-OHP group, 35.33 \pm 6.15 days; 5-FU group, $48.83 \pm 4.54$ days; TAX+Apatinib group, $60.67 \pm 20.70$ days; $\mathrm{L}-\mathrm{OHP}+$ Apatinib group, $40.00 \pm 8.39$ days; and 5-FU+Apatinib group, $46.83 \pm 7.03$ days. The survival time of L-OHP monotherapy and combination groups decreased obviously; a significant difference existed when the 2 groups were compared with the control group $(P<0.05$; Figure 2). The combination of 2 other chemotherapy drugs with Apatinib had minimal effect on the survival time (Figure 2). According to the former pharmacodynamics experiment results, we concluded that the combination of Apatinib and L-OHP was not appropriate, and the combination may have increased the toxicity.

\section{Apatinib combined with chemotherapy decreases the MVD}

The MVD of the monotherapy groups was as follows: Apatinib group, 15.00 $\pm 2.00 /$ per view field; TAX group, $13.66 \pm 2.08 /$ per view field; L-OHP group, 16.33 $\pm 2.51 /$ per view field; 5-FU group, 17.00 \pm 1.00 /per view field; and control group, 20.66 $\pm 2.08 /$ per view field. The differences were significant when the medication group was compared with the control group $(P<0.05)$. The MVD of the combination groups was as follows: TAX+Apatinib group, $9.33 \pm 0.58 /$ per view field; L-OHP+Apatinib group, 9.66 $\pm 1.15 /$ per view field; and 5-FU+Apatinib group, 11.00 $\pm 1.73 /$ per view field. A significant difference was observed when comparing the chemotherapy monotherapy group and the control group $(P<0.05$; Figure 2).

\section{Discussion}

The prognosis of gastric cancer is closely related with the time of treatment. At the early stage, patients can receive radical excision during the endoscopy, and the 5-year survival rate is $>90 \%$. However, most of the patients in our country are diagnosed at the late stage or have distant metastasis; for these patients, systemic therapy (mainly chemotherapy) is still the best choice for advanced gastric cancer patients, but the development of chemotherapy for gastric cancer comes into the bottleneck stage and the improvement of survival time is extremely limited. In 2016, the National Comprehensive Cancer Network (NCCN) guidelines for gastric cancer ${ }^{22}$ recommended that platinum, taxane, and other drugs are still the first-line chemotherapy regimen recommendation, but due to the limited clinical efficacy of single drug and $<9$ month improvement for the OS, combination regimens (such as FP/XP and FOLFOX/XELOX) are becoming the main recommendation for first-line treatment. Although the triple regimens could improve the OS to some extent, under the new guidelines, their increased toxicity relegates such regimens to being only alternative treatments. Without obvious alteration, docetaxel, TAX, and irinotecan are still the main representatives for second-line chemotherapy, but for treatment beyond the second line, no standard chemotherapy regime is available for us to consult. Therefore, more effective treatments with low toxicity are required for clinics to improve the survival time of patients with advanced gastric cancer.

With the progress of genomics, the importance of targeted drugs in the treatment of gastric cancer has become increasingly prominent. Trastuzumab combined with chemotherapy $^{23}$ prolonged the OS of the human epidermal growth factor receptor 2 (HER2)-positive advanced gastric cancer patients (IHC $3+$ or IHC $2+$ FISH+) to 16.0 months, almost 5 months longer than the monotherapy group. Based on this result, trastuzumab has become the first choice for gastric cancer patients with HER2-overexpression according to the NCCN guidelines. Many clinical trials showed that the rate of HER2-positive gastric cancers was only $7.3 \% \sim 20.2 \%$; so, only few people can benefit from antiHER2 treatment. Moreover, other targeted drugs also demonstrate some progress, such as the inhibitors of the VEGF/VEGFR angiogenic signaling pathway. For example, ramucirumab is a monoclonal antibody that targets VEGFR-2 and exhibits the ideal effect in the second-line treatment for advanced gastric cancer. In the RAINBOW trial, the OS was prolonged to 9.6 months when ramucirumab was combined with taxol, 2.2 months longer than the taxol monotherapy group. ${ }^{24}$ At the same time, the new TKI Apatinib, ${ }^{11}$ which also acted on the same target, led to a big breakthrough in the field of gastric cancer beyond the second-line treatment, providing more curative opportunities for the patients who failed in multiple lines of chemotherapy. In this study, the survival time was obviously prolonged for the Apatinib group $(n=181), 1.8$ months longer than the control group $(n=92$; hazard ratio $[\mathrm{HR}]=0.709, P=0.0149$ ); the progress-free time of the medication group was also prolonged (2.6 months vs 1.8 months, $\mathrm{HR}=0.444, P<0.0001$ ), and quality of life 
was improved. Apatinib is a new, small-molecule TKI that was developed independently; it can highly and selectively inhibit the tyrosine kinase activity of VEGFR-2 and block the downstream signal conduction, thus inhibiting tumor angiogenesis and tumor growth. Previous studies ${ }^{25,26}$ suggested that anti-angiogenic drugs are effective in inhibiting tumor growth by reducing the number of vessels in the tumor, destroying vascular function, and cutting off the oxygen and nutrient supply, mechanisms that are different from the cytotoxic effects of the drugs. Apatinib showed an obvious anti-tumor character in the previous studies, especially in the inhibition of cell growth and promotion of apoptosis in some in vitro experiments. In our experiment, Apatinib monotherapy, which has a broad spectrum of anti-tumor effects, could inhibit the invasion and migration ability of gastric cancer cells, inhibit proliferation, and promote apoptosis. These results are consistent with existing literature.

There are different mechanisms for anti-angiogenic drugs and cytotoxic chemotherapy drugs. ${ }^{17}$ The first act on the tumor microenvironment to degenerate the existing tumor vasculature or inhibit the growth of tumor vasculature. In addition, anti-angiogenic drugs can improve the oxygen content and increase the concentration of chemotherapy drugs in the tumor by normalizing the internal vessels of the tumor and repairing the abnormal tumor vascular system. The second mechanism aims to directly kill the tumor cells and finally inhibit the tumor growth. Additionally, some studies showed that small doses of cytotoxic chemotherapy drugs can also have the anti-angiogenic effects. ${ }^{27}$ Therefore, the advantage of clinical application is obvious when cytotoxic drugs are combined with anti-angiogenic drugs for overlapping reactions.

In this experiment, we chose classical first-line treatment drugs for gastric cancer, TAX, L-OHP, and 5-FU, combined with Apatinib to investigate whether the inhibition activity increased. We found that anti-tumor activity increased after the combination, but obvious differences still existed in the in vitro and in vivo experiments. The reasons are as follows: first, we applied statistical $P$-values to check the statistical significance and the CDI index to evaluate the degree of the combination. ${ }^{28}$ In the cytotoxicity test, we found statistical significance $(P<0.05)$ in every combination group compared with the corresponding chemotherapy group. For the CDI index, only TAX combined with Apatinib showed obvious synergic effects; the synergic effect was not obvious for L-OHP and 5-FU. We fixed the concentration of chemotherapy drugs at a sufficiently low level to elicit minimal cytotoxicity and then combined them with different concentrations of Apatinib. We assumed that low concentrations of the 2 drugs may have the ability to improve the drug effect beyond additive effects; that means " $1+1>2$," or we can say that it has a synergistic effect. When the concentration of Apatinib increased to a certain level, the cytotoxicity effect was strong enough to kill the cell, but there was not much room for improvement of the combination effect; that means " $2>1+1>1$," or we can say that it has an antagonistic effect.

Therefore, we assumed that the 2 indexes focus on different aspects, which are not contradictory for the conclusion. Second, we fixed the cell line and drug dosage for further analysis of the mechanism based on the cytotoxicity test result; the synergic effect was much more obvious for the combinations with Apatinib $4 \mu \mathrm{g} / \mathrm{mL}$. In the apoptosis assay, the combination of 5-FU with Apatinib increased the apoptosis ratio; in addition, the ratio of the corresponding effector proteins $\mathrm{Bax} / \mathrm{Bcl}-2$ increased, and the protein expression of c-caspase-3, which is the most important apoptotic executive protein, increased obviously in the 5-FU+Apatinib group compared with that of the 5-FU group. The trend of this result was consistent with the flow cytometry analysis, but the same apoptosis ratio with the addition of TAX and L-OHP changed minimally. Thus, we assumed that the proapoptosis effect played an important role in the combination of 5-FU and Apatinib. In the invasion and migration assays, we found statistical significance $(P<0.05)$ in every combination group compared with the corresponding chemotherapy groups; thus, we assumed that inhibition of cell invasion and migration is universally relevant in combination of the 3 chemotherapy drugs and Apatinib. In the xenograft model test, the inhibition ability increased when TAX and 5-FU combined with Apatinib; additionally, the volume and the fluorescence brightness of the tumors changed minimally in the fluorescence imaging system. For the L-OHP combination group, the increased tumor volume and the strengthened fluorescence brightness showed that this combination is inappropriate. This result was also verified by the subsequent MVD test; L-OHP combined with Apatinib did not obviously decrease the MVD, and no statistical significance was found between the groups $(P>0.05)$. As a conclusion, we can say that certain individual differences exist in the combination of chemotherapy drugs and Apatinib, although the general trend showed that the inhibition effect was enhanced after the combination. However, only TAX and 5-FU are proper for the combination with less toxicity.

Based on the above results, we can assume that the antitumor activity is enhanced when Apatinib is combined with classical first-line chemotherapy drugs for gastric cancer. 
Although the best mode of combination and dosing of the drugs still requires additional experiments and further confirmation, the synergistic effect of the combination was obvious. It is believed that with the development of Apatinib-related experiments, this treatment strategy will be increasingly used in more areas for cancer treatment and will benefit more cancer patients in the future.

\section{Disclosure}

The authors report no conflicts of interest in this work.

\section{References}

1. Malvezzi M, Bertuccio P, Rosso T, et al. European cancer mortality predictions for the year 2015: does lung cancer have the highest death rate in EU women? Ann Oncol. 2015;26:779-786.

2. Rebecca LS, Kimberly DM, Ahmedin J. Cancer Statistics, 2015. CA Cancer J Clin. 2015;65:25-29.

3. Chen WQ, Zheng RS, Peter D, et al. Cancer Statistics in China 2015. CA Cancer J Clin. 2016;66:115-132.

4. Salah-Eddin A, Joerg TH, Stephan P, et al. Phase III trial in metastatic gastroesophageal adenocarcinoma with fluorouracil, leucovorin plus either oxaliplatin or cisplatin: a study of the arbeitsgemeinschaft internistische onkologie. J Clin Oncol. 2008;26(9):1435-1441.

5. Kang YK, Kang WK, Shin DB, et al. Capecitabine/cisplatin versus 5-fluorouracil/cisplatin as first-line therapy in patients with advanced gastric cancer: a randomized Phase III noninferiority trial. Ann Oncol. 2009;20(4):666-673.

6. Van CE, Moiseyenko VM, Tjulandin S, et al. Phase III study of docetaxel and cisplatin plus fluorouracil compared with cisplatin and fluorouracil as first-line therapy for advanced gastric cancer: a report of the V325 Study Group. J Clin Oncol. 2006;24(31):4991-4997.

7. Wagner AD, Unverzagt $\mathrm{S}$, Grothe W, et al. Chemotherapy for advanced gastric cancer. Cochrane Database Syst Rev. 2010;3:CD004064.

8. Cervantes A, Roda D, Tarazona N, Roselló S, Pérez-Fidalgo JA. Current questions for the treatment of advanced gastric cancer. Cancer Treat Rev. 2013;39:60-67.

9. Lordick F. Unmet needs and challenges in gastric cancer: the way forward. Cancer Treat Rev. 2014;40(6):692-700.

10. Power DG, Kelsen DP, Shah MA. Advanced gastric cancer slow but steady progress. Cancer Treat Rev. 2010;36(5):384-392.

11. Li J, Qin S, Xu JM, et al. Randomized, double-blind, placebo-controlled Phase III Trial of Apatinib in patients with chemotherapy-refractory advanced or metastatic adenocarcinoma of the stomach or gastroesophageal junction. J Clin Oncol. 2016;34(13):1448-1454.

12. Pei K, Ji X, Li HJ, et al. Advanced gastric cancer treated by apatinib: one case report. J Chinese Oncol. 2016;22(4):336-338.

13. Du J, Liu BR. Clinical observation of Apatinib in the treatment of chemotherapy resistant advanced gastric cancer. Mod Oncol. 2016; 24(13):2100-2104.
14. Zhou YY, Qin SK, Wang R, et al. Four cases of the Apatinib in the treatment of advanced gastric cancer with intractable malignant ascites. Chin Clin Oncol. 2016;21(5):476-478.

15. Wang PY, Shang NN, Liu Z, et al. The clinical research of Apatinib combined with Tegafur in the second line treatment of the advanced gastric cancer. J Taishan Med Col. 2016;37(8):919-920.

16. Zhu XR, Zhu ML, Wang Q, et al. A case report of targeted therapy with apatinib in a patient with advanced gastric cancer and high serum level of alpha-fetoprotein. Medicine. 2016;95:37:e4610.

17. Sitohy B, Nagy JA, Dvorak HF. Anti-VEGF/VEGFR therapy for cancer: reassessing the target. Cancer Res. 2012;72(8):1909-1914.

18. Martin R, Rolf K, Anders M, et al. Docetaxel plus Nintedanib versus docetaxel plus placebo in patients with previously treated non-smallcell lung cancer (LUME-Lung 1): a phase 3, double-blind, randomised controlled trial. Lancet Oncol. 2014;15:143-155.

19. Wang DG, Wang ZP, Tian BQ, Li X, Li S, Tian Y. Two-hour exposure to sodium butyrate sensitizes bladder cancer to anticancer drugs. Int $J$ Urol. 2008;15:435-441.

20. Weidner N. Current pathologic methods for measuring intratumoral microvessel density within breast carcinoma and other solid tumors. Breast Cancer Res Treat. 1995;36(2):169-180.

21. Li J, Zhao XM, Chen L, et al. Safety and pharmacokinetics of novel selective vascular endothelial growth factor receptor-2 inhibitor YN968D1 in patients with advanced malignancies. BMC Cancer. 2010; 10:529-536.

22. Ajani JA, D'Amico TA, Almhanna K, et al. Gastric cancer, version 3. 2016, NCCN clinical practice guide lines in oncology. J Natl Compr Canc Netw. 2016;14(10):1286-1312.

23. Yung JB, Eric VC, Andrea F, et al. Trastuzumab in combination with chemotherapy versus chemotherapy alone for treatment of HER2-positive advanced gastric or gastro-oesophageal junction cancer (ToGA): a phase 3, open label, randomised controlled trial. Lancet. 2010;376:687-697.

24. Hansjochen W, Kei M, Eric VC, et al. Ramucirumab plus paclitaxel versus placebo plus paclitaxel in patients with previously treated advanced gastric or gastro-oesophageal junction adenocarcinoma (RAIN BOW): a double blind, randomised phase 3 trial. Lancet Oncol. 2014; 15(11):1224-1235.

25. Lin Y, Zhai ET, Liao B, et al. Autocrine VEGF signaling promotes cell proliferation through a PLC-dependent pathway and modulates Apatinib treatment efficacy in gastric cancer. Oncotarget. 2017;8(7): 11990-12002.

26. Zhong CY. Apatinib, a New Small Molecular VEGFR2 Inhibitor, Suppresses the Activity of Lung Cancer Stem Cells [EB/OL]. Available from: http://wclc.ioncol.com/newsview-2233-771-0.html. Accessed December 9, 2016.

27. Norrby K. Metronomic chemotherapy and anti-angiogenesis: can upgraded pre-clinical assays improve clinical trials aimed at controlling tumor growth? APMIS. 2014;122(7):565-579.

28. Chou TC. Theoretical basis, experimental design, and computerized simulation of synergism and antagonism in drug combination studies. Pharmacol Rev. 2006;58(3):621-681.
OncoTargets and Therapy

\section{Publish your work in this journal}

OncoTargets and Therapy is an international, peer-reviewed, open access journal focusing on the pathological basis of all cancers, potential targets for therapy and treatment protocols employed to improve the management of cancer patients. The journal also focuses on the impact of management programs and new therapeutic agents and protocols on

Submit your manuscript here: http://www.dovepress.com/oncotargets-and-therapy-journal

\section{Dovepress}

patient perspectives such as quality of life, adherence and satisfaction. The manuscript management system is completely online and includes a very quick and fair peer-review system, which is all easy to use. Visit http://www.dovepress.com/testimonials.php to read real quotes from published authors. 